Investigación

\title{
ROL DEL ESTUDIANTE EN LOS ESCENARIOS DE PRÁCTICA Y BENEFICIOS DE LAS INSTITUCIONES INTERVENIDAS POR LOS ESTUDIANTES DE VII SEMESTRE, DE LA UNIVERSIDAD CATÓLICA DE MANIZALES 2017- 2019, COLOMBIA
}

ROLE OF THE STUDENT IN THE PRACTICE SCENARIOS AND BENEFITS OF THE INSTITUTIONS INTERVENED BY THE STUDENTS OF VII SEMESTER OF THE CATHOLIC UNIVERSITY OF MANIZALES 2017- 2019, COLOMBIA

\section{Nancy Álvarez Castaño}

Enfermera especialista en Administración de Servicios de Salud

Universidad Católica de Manizales, Manizales, Colombia

nanalvarez@ucm.edu.com

https://orcid.org/0000-0001-6878-8031

\section{Joan Valentina Ocampo}

Estudiante de enfermería

Universidad Católica de Manizales, Manizales, Colombia

Joan.ocampo@ucm.edu.com

\section{Manuela Cadavid}

Estudiante de enfermería

Universidad Católica de Manizales, Manizales, Colombia

Manuela.cadavid@ucm.edu.com

Artículo recibido el 15 de junio de 2021. Aceptado en versión corregida el 26 de agosto de 2021.

\section{RESUMEN}

El programa de enfermería de la Universidad Católica de Manizales (Colombia) incluye escenarios prácticos en los cuales se desarrollan, entre otros, la ejecución de planes de mejoramiento que buscan fortalecer en los estudiantes competencias relacionadas al quehacer profesional, al mismo tiempo que se busca apoyar las Instituciones Prestadoras de Servicios de Salud (IPS) en el cumplimiento de sus funciones, en respuesta a las necesidades de estas instituciones. OBJETIVO: Identificar el rol del estudiante de VII semestre en los planes de mejoramiento realizados en las IPS, durante las prácticas formativas del programa de enfermería de la Universidad Católica de Manizales entre los años 2017 y 2019. MÉTODOS: Se realizó un estudio descriptivo retrospectivo de tipo documental, que permitió la revisión del archivo de 190 planes de mejoramiento realizados por este grupo de estudiantes, en el periodo definido. Las variables de estudio fueron: Grupos poblacionales, prácticas clínicas, educativas y administrativas. Se realizó análisis descriptivo de base de datos en Excel de 
Rol del estudiante en los escenarios de práctica y beneficios de las instituciones... acuerdo con las variables definidas en el instrumento. RESULTADOS: Dentro de las prácticas realizadas por parte de los estudiantes la más frecuente fue la de tipo clínico, seguida de administrativa y promoción de la salud, realizadas en áreas de urgencias, hospitalización, consulta externa, quirófano, unidad de cuidados intensivos y área administrativa. Con respecto a la población beneficiaria de las intervenciones, el mayor número estuvo en el grupo de adultos (29 - 59 años) en quienes el tópico más abordado fue el autoexamen de mama y citología, de acuerdo a las necesidades institucionales. Dentro de las actividades administrativas y asistenciales más ejecutadas, se encuentran pausas activas y manejo de corto punzantes, respectivamente. En relación a la capacitación se tiene que se benefició un total de 1226 usuarios internos y 1703 usuarios externos. CONCLUSIÓN: El rol que desempeñan los estudiantes de VII semestre es de gran importancia para apoyar y apalancar procesos administrativos, clínicos, de promoción y mantenimiento de la salud en las IPS, debido a que permiten incrementar la fuerza de respuesta, además ofrece la oportunidad para aprovechar este talento humano en beneficio de educación para cliente interno y externo, identificación de problemas y según los resultados; diseñar y hacer seguimiento a los procesos que en su momento se observan como críticos.

Palabras clave: prácticas formativas, educación, salud.

\section{ABSTRACT}

The nursing program of Universidad Católica de Manizales (Colombia) includes practical scenarios in which it developments, among others, the execution of improvement plans for the strengthen in students the skills related to professional work, while searching to support to the health providing institutes on theirs functions, in response to the institutional needs. OBJECTIVE: To identify the VII semester student's role in improvement plans made on health providing institutes, during formatives practices of nursing program of Universidad Católica de Manizales since 2017 until 2019. METHODS: A descriptive retrospective documental kind study was made, that allowed the revision of the 190 improvement plan's file who carried out by this group of students, in the defined period. The variables were: population groups, clinical, educational and administrative practices. Descriptive analysis of Excel data base was made, according the defined variables. RESULTS: The most common practice made for this groups of students were clinical kind, followed by administrative and health promotion, this practices were made in emergency, hospitalization, external consultation, surgery, intensive care unit, and administrative areas. With respect to beneficiary population of the interventions, the greater number was in group of adults (2959 years old) in whom the most approached issue was breast self-examination and cytology, in response to institutional needs. Within the most frequent clinical and administrative activities were active breaks and sharp tools management, respectively. In relation to training, it benefices 1226 internal users and 1703 users of health services. CONCLUSION: the VII semester student's role is of great importance to support and leverage administrative, clinical, promotion and maintenance processes of health in the health providing institutes, because they provide an increase in the response force, it also offers the opportunity to take advantage 
of this human talent for the benefit of education for internal and external users of this services, identification of problems and according to the results, design and monitor the processes that are observed at the time as critics.

Keywords: training practices, education, health.

http://dx.doi.org/10.7764/Horiz_Enferm.32.2.155-176

\section{INTRODUCCIÓN}

En la formación del personal de salud, es indispensable su interacción con las instituciones prestadoras de servicios de salud (IPS), es por ello que se debe contar con un entorno de práctica que brinde la posibilidad a los estudiantes de desarrollar sus competencias teóricoprácticas adquiridas durante el proceso de formación académica, y también es compromiso de ellos prestar un servicio de calidad mientras se encuentren en sus lugares de práctica, desempeñando su rol como futuros enfermeros de una manera responsable y basados en los conocímientos adquiridos.

Durante la formación de los profesionales en enfermería, una de las actividades que apoya al estudiante para el logro de competencias son las asignaturas prácticas, las cuales están enfocadas en los diferentes niveles de atención y grupos poblacionales, desempeñando su labor en la comunidad y en el entorno hospitalario; a su vez en los estudiantes generan criterios de responsabilidad en cuanto a sus acciones y cuidado de los pacientes. En este sentido, Figueiredo et al., en el artículo "Adverse events related to pratical assistence: an integrative review" expresa que la seguridad de los pacientes en este entorno hospitalario es una responsabilidad netamente del profesional (equipos médicos y de enfermería), exigiendo que los estudiantes desde sus prácticas formativas y teóricas garanticen a sus pacientes un trato seguro y sobre todo una calidad en la atención ${ }^{1}$.

Igualmente, Tirado et al., afirman que “(..) el profesional de enfermería, tiene el perfil para trabajar la estrategia de Atención Primaria en Salud (APS) en el ámbito comunitario" ${ }^{2}$. Es por eso qué en el último año del programa, los estudiantes de VII semestre de la Universidad Católica de Manizales, enfocan su educación en gestiones administrativas, donde adquieren habilidades para el desarrollo de las intervenciones de promoción y mantenimiento de la salud a nivel individual, familiar y comunitario. A su vez, de acuerdo con Mármol, et al. se encuentra que "la enfermera se funda como gestora y coordinadora de casos, así como reguladora de flujos asistenciales y referente de los pacientes con problemas de salud para el seguimiento de sus procesos, la activación de recursos y, en programas de autocuidado de pacientes activos o expertos, favoreciendo el empoderamiento de las personas" 3 .

Las iniciativas y las estrategias que se implementan, garantizan la atención primaria en salud, el tratamiento y rehabilitación de los individuos, familias y 
Rol del estudiante en los escenarios de práctica y beneficios de las instituciones...

comunidades dentro del campo clínico, externo y administrativo siendo algunas de estas intervenciones: priorizar, acompañar, inducir, identificar, remitir y apoyar las necesidades en salud de la población en específico. Para garantizar la calidad de la atención en salud, Escobar, et al; expresa que: "los profesionales de enfermería tienen como fin dar cuidado integral de salud a la persona, a la familia, la comunidad y a su entorno" ${ }^{\text {. Estos son }}$ aspectos importantes para el rol del futuro profesional de enfermería, porque las prácticas formativas propician el perfeccionamiento de destrezas y aprendizajes, en particular los estudiantes de VII semestre que despliegan actividades administrativas y asistenciales, generando en ellos habilidades que les pueden ayudar a desempeñarse en su rol profesional con énfasis en humanización y de atención con calidad a sus pacientes.

Ahora bien, según Guerrero et al., el cuidado humanizado de enfermería, de acuerdo a la teoría de Jean Watson, servicio de medicina del Hospital Daniel Alcides Carrión, expresa que "el Cuidado Humanizado es una necesidad que urge en la práctica profesional y esto se evidencia en las políticas, disposiciones normativas establecidas por las entidades de salud que velan por el derecho de los pacientes y garantizan la calidad del servicio que se brinda"5, generando una inclusión de la Política de Atención Integral en Salud (PAIS) de Colombia, la cual define ésta como estrategias e instrumentos que permiten alcanzar objetivos de un sistema centrado en la población y sus relaciones a nivel familiar y comunitario, reconociendo que los problemas de salud son generados o potenciados por las condiciones ambientales, sociales, culturales, políticas, económicas, educacionales, de hábitat y genéticos que afectan a las poblaciones en los diferentes ámbitos territoriales que conforman la nación ${ }^{6}$.

En este orden de ideas, este trabajo es importante porque visibiliza la relación efectiva entre las Instituciones de Educación Superior y las instituciones de salud (IPS) que ofertan los escenarios de práctica; lo cual permite el empoderamiento del rol del estudiante de enfermería, el cual tiene la oportunidad de intervenir en los procesos de mejoramiento, de calidad en la atención en salud y acreditación institucional.

Adicionalmente, investigar cómo se desenvuelve el estudiante de VII semestre en las prácticas formativas, y cómo se benefician las IPS con los planes de mejoramiento que estos estudiantes han dejado en las diferentes rotaciones es relevante para el programa debido a que demuestra la actuación de ellos en su qué hacer durante estas prácticas y la trascendencia a nivel local y nacional.

Es importante analizar en estudiantes universitarios del área de enfermería la relación entre el proceso cognitivo y práctico, en busca de aproximar nuevas miradas para dar respuesta, de algún modo, a las demandas sociales que enfrenta actualmente el sistema de salud, dado que se requieren profesionales competentes para desarrollarse en entornos cambiantes. Por lo tanto, es importante visibilizar al futuro egresado con un desempeño eficaz en los diferentes ámbitos profesionales.

Por este motivo se plantea el objetivo de identificar el rol del estudiante de VII semestre en los planes de 
mejoramiento realizados en las IPS, durante las prácticas formativas del programa de enfermería de la Universidad Católica de Manizales entre los años 2017 y 2019.

\section{METODOLOGÍA}

Se realizó un estudio descriptivo retrospectivo de tipo documental, que permitió la revisión en el archivo central de la Universidad Católica de Manizales, de los planes de mejora realizados por estudiantes de enfermería de VII en el periodo comprendido de enero del 2017 a diciembre del 2019. En la tabla 1 se observan las variables que se tuvieron en cuenta para la revisión de los planes de mejora.

Tabla 1. Variables revisadas en los planes de mejoramiento

\begin{tabular}{|c|c|c|c|}
\hline Grupos & Clínicas & Educativas & Administrativas \\
\hline Instituciones & IASS & Usuarios internos & Políticas \\
\hline caracterizadas & Seguridad del & Usuarios externos & institucionales \\
\hline Usuarios & paciente & Autocuidado & Calidad en \\
\hline beneficiados & Protocolos & Promoción de la & atención \\
\hline Programas & institucionales & salud & \\
\hline intervenidos & Población de riesgo & & \\
\hline Servicios & & & \\
\hline beneficiados & & & \\
\hline Grupos por curso de & & & \\
\hline vida & & & \\
\hline
\end{tabular}

Fuente: Elaboración propia

Se realiza un análisis cuantitativo de estas variables, además que se realiza análisis interpretativo de cada uno de los resultados, para definir así el rol de los estudiantes dentro de cada uno de estos resultados y lograr así el cumplimiento del objetivo, a través de aspectos como participación, desarrollo de competencias, desarrollo de temáticas, población beneficiaria, apoyo a compromisos institucionales, entre otros.

Se excluyeron aquellos documentos que no contaban con la información completa requerida para desarrollar el trabajo. Se realizó análisis descriptivo de base de datos en Microsoft
Excel de acuerdo con las variables definidas en el instrumento.

\section{RESULTADOS}

Los resultados obtenidos se han estructurado según los criterios seleccionados para la identificación del rol del estudiante, los procesos abordados por ellos en las IPS y las personas que se benefician de las prácticas, los resultados de los planes de mejoramiento analizados en este proyecto fueron todos ejecutados por parte de los estudiantes de VII semestre de enfermería de la Universidad Católica de Manizales. Se aplicaron los criterios, según las variables definidas, a 
Rol del estudiante en los escenarios de práctica y beneficios de las instituciones... los documentos encontrados en los minados de aprendizaje; a su vez las accioarchivos para la realización del análisis y valoración de los resultados frente a otras fuentes consultadas.

En los documentos se pueden apreciar los trabajos de los planes de mejoramiento y las actividades realizadas, que dan fe sobre el desempeño y las competencias alcanzadas, capacidades y habilidades de acuerdo a los niveles deternes desarrolladas y acompañamiento de la IPS por parte de la universidad.

Los resultados que se muestran a continuación, evidencian el trabajo de los estudiantes de VII semestre en el rol clínico, administrativo y educativo en la promoción y mantenimiento de la salud (Figura 1).

Figura 1. Tipos de práctica realizada por los estudiantes de VII semestre de enfermería. Universidad Católica de Manizales. 2017-2019. Fuente. Elaboración propia

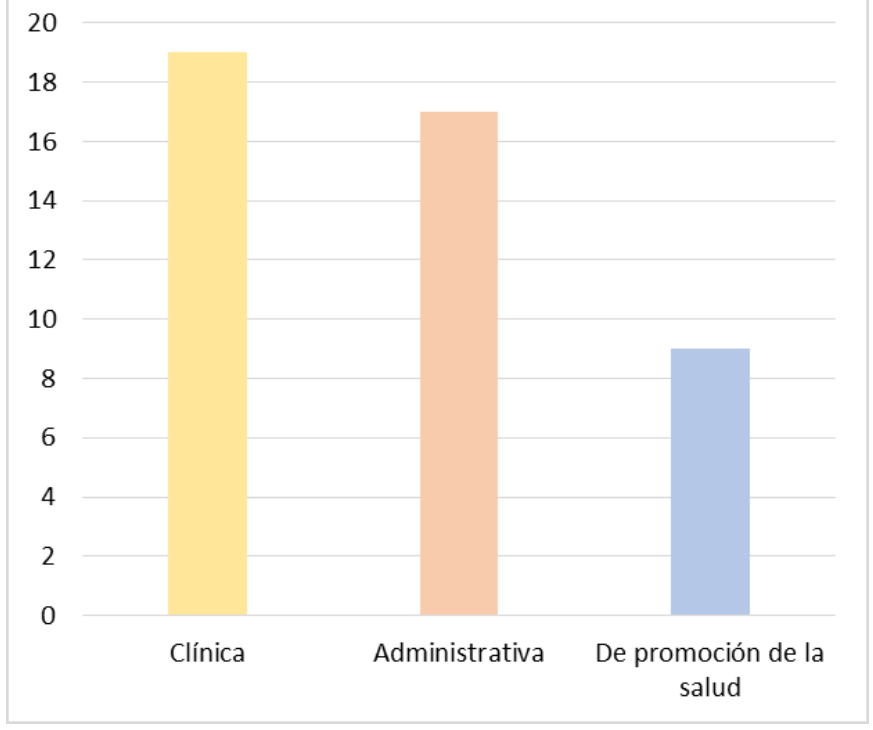

En la Figura 1 se observa la distribución de las prácticas que los estudiantes de séptimo semestre desarrollan según las actividades que desempeñan en las diferentes entidades clínicas (públicas o privadas), así:

- La práctica clínica es asistencial orientada hacia la promoción y detección temprana de la enfermedad, intervención en el tratamiento, rehabilitación, y recuperación de la salud con énfasis en el cuidado de la vida y la salud humana.
- La orientación de la práctica administrativa tiene que ver con apoyo procesos gerenciales, de coordinación, de manejo de personal, capacitación de cliente interno y externo, apoyo $y$ seguimiento a políticas institucionales de calidad y seguridad de paciente entre otros. El énfasis de este semestre es el área de administración y gerencia de los servicios de salud, de los servicios de enfermería y al desarrollo de planes de mejoramiento en salud relacionados con epidemiología, calidad, seguridad 
del paciente, direcciones científicas; áreas de consulta externa y hospitallización. Los estudiantes diferentes instituciones como: entes de control, instituciones de salud de baja, mediana y alta complejidad ubicadas en los departamentos de Caldas, Quindío, Valle y Antioquia.

- Los escenarios de práctica comunitarios están orientados hacia la atención primaria en salud (APS) así como la promoción de la salud, que constituye la práctica de promoción de la salud. Esta se despliega en los diferentes ámbitos en donde la persona vive, estudia, trabaja, se recrea y se desarrolla.

Cabe aclarar que el estudiante también puede participar de programas institucionales del ámbito clínico que también están enfocados a diferente tipo de actividades que están en beneficio general de los servicios (Figura 2).

Figura 2. Servicios beneficiados por los planes de mejoramiento, elaborados por estudiantes de VII semestre de enfermería. Universidad Católica de Manizales. 2017-

2019. Fuente. Elaboración propia.

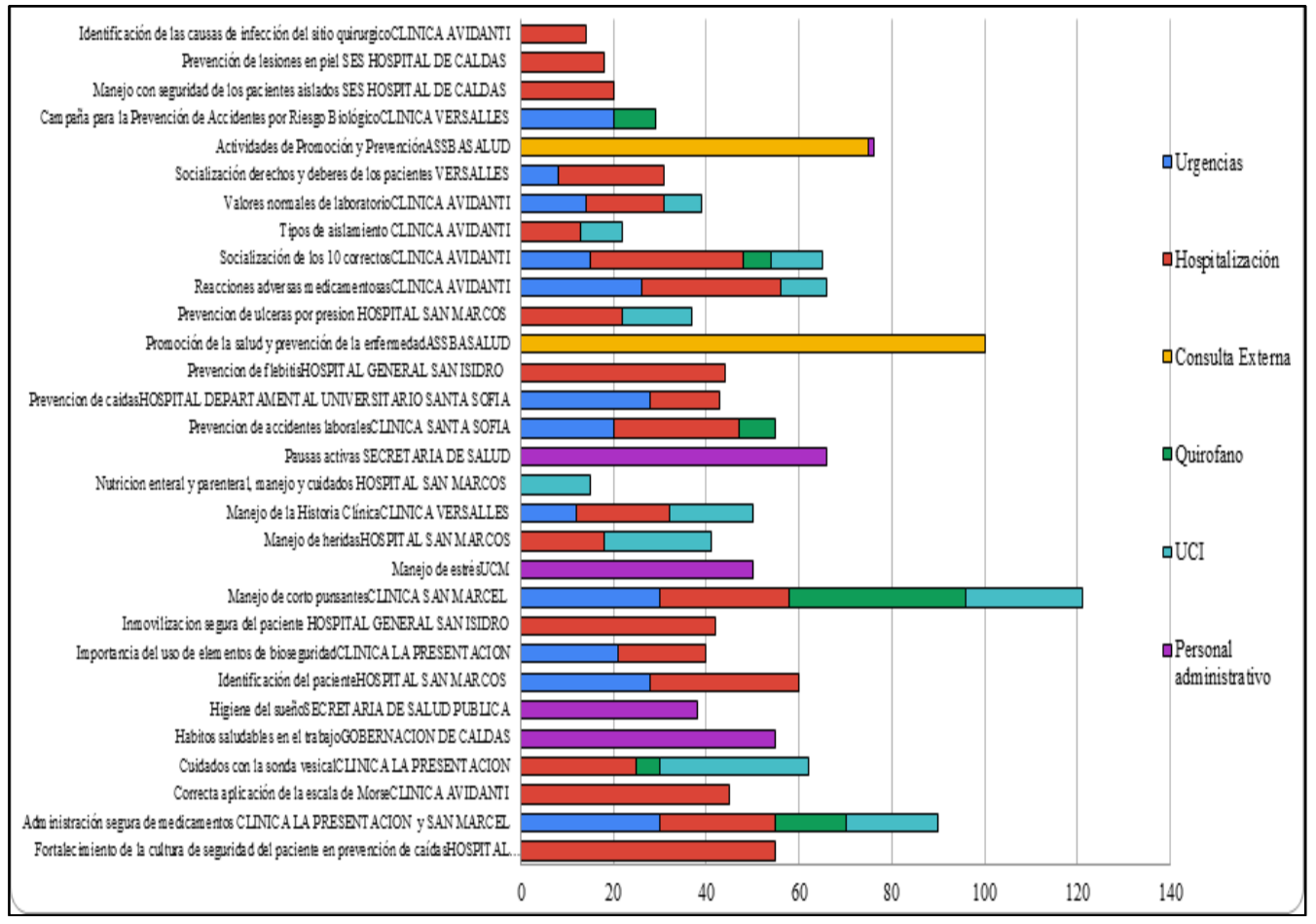

Para los estudiantes objeto de estudio los escenarios político, social, cultural, ambiental, económico y productivo de las IPS y comunidades son el quehacer natural en las prácticas, con el propósito de mejorar estos aspectos se incluyen tópicos como: rendimiento, productividad, desarrollo humano, robustecimiento de prácticas culturales de autocuidado en la comunidad, prevención de la enfermedad, mejora en las condiciones de vida, entre otros.

En el momento de desglosar las actividades por plan de mejora se 
Rol del estudiante en los escenarios de práctica y beneficios de las instituciones...

evidencia no solo lo anteriormente descrito, además se observan también la labor en educación, prevención y mantenimiento de la salud, acciones de calidad y aseguramiento de las políticas institucionales como: prevención y manejo del evento adverso, programa de garantía de calidad en salud y seguridad del paciente. En el gráfico 2 se pudieron identificar los dos planes de mejora que más servicios integraron:

- Manejo de corto punzante realizado en la Clínica San Marcel, integrando a los servicios de: hospitalización, urgencias, quirófano y Unidad de Cuidados Intensivos (UCI), con un total de 120 personas beneficiadas por todos los servicios.

- Administración segura de medicamentos el cual se llevó a cabo en la Clínica de la Presentación y la Clínica San Marcel, integrando los servicios de: urgencias, hospitalización, quirófano y UCI, con un total de 90 personas beneficiadas por todos los servicios.

Esto permite evidenciar como el estudiante de enfermería tiene un propósito institucional en el rendimiento y productividad, cumplimiento de norma y aseguramiento de la atención para el paciente, a través del desarrollo de este tipo de actividades.

En relación a los servicios beneficiados por los planes de mejora, se identificó:

- En el servicio de urgencias, el plan de mejora con mayor impacto en la población fue el de manejo de corto punzante y administración segura de medicamentos con un total de 30 usuarios internos beneficiados por cada uno. Por otro lado, la socialización de derechos y deberes del usuario, con un total de 8 usuarios internos beneficiados, tuvo el menor impacto.

- En el servicio de hospitalización, el plan de mejora con mayor impacto en la población fue fortalecimientos de la política de seguridad del paciente en caídas con un total de 55 usuarios internos beneficiados, mientras que el que menor impacto estuvo en el plan de mejora relacionado al tipo de aislamiento, con un total de 13 usuarios internos beneficiados.

- En el servicio de consulta externa, el plan de mejora con mayor impacto en la población fue el de promoción de la salud y prevención de la enfermedad, con un total de 100 usuarios internos beneficiados, contrario al de actividades de promoción y prevención con un total de 75 usuarios internos beneficiados.

- En el servicio de quirófano, el plan de mejora con mayor impacto en la población fue el de manejo de corto punzante con un total de 38 usuarios internos beneficiados, mientras que el de cuidados con la sonda vesical impactó 5 usuarios internos beneficiados.

- En el servicio de UCI, el plan de mejora con mayor impacto en la población fue el de manejo con la sonda vesical con un total de 32 usuarios internos beneficiados. Por otro lado, el menor impacto estuvo en el plan de de valores normales de laboratorio con un total de 8 usuarios internos beneficiados.

- En cuanto al personal administrativo, el plan de mejora con mayor impacto fue el de pausas activas con un total de 66 
Álvarez Castaño N, Ocampo JV, Cadavid M

usuarios internos beneficiados.

Contrario a esto, el de promoción y prevención con una sola persona beneficiada, se considera la de menor impacto.

Finalmente, el total de población beneficiada por planes de mejora en los servicios de urgencias, hospitalización, consulta externa, quirófano, UCI y personal administrativo fue de 1.527 personas.

Para el estudio, también se realizó un análisis del número de personas que fueron beneficiadas por cada IPS donde se llevaron a cabo los planes de mejoramiento descritos. A continuación, se presentan las instituciones con las cuales se realizan las prácticas objeto de esta investigación:

1. Las instituciones prestadoras de salud en la que mayor población se vio beneficiada por los planes de mejora realizados fueron:

- Los colegios de Manizales y Villanería en un programa que trabajan con conjunto con la con la Dirección Territorial de Salud de Caldas con un total de 1.022 personas beneficiadas.

- El Hospital San Jorge de Pereira con un total de 702 personas beneficiadas.

- El Hospital de San José de Neira con un total de 86 personas beneficiadas

2. Las instituciones prestadoras de salud en la que menor población participó fueron:

- La Clínica de la Presentación con un total de 5 personas beneficiadas

- La Clínica del Rosario con un total de 7 personas beneficiadas

- El Hospital San Cayetano y el Hospital Infantil de la cruz roja con un total de 15 personas beneficiadas.

Esta información se presenta en la Figura 3.

Figura 3. Grupos e Institución beneficiada donde se realizaron las prácticas, elaboradas por estudiantes de VII semestre de enfermería. Universidad Católica de Manizales. 20172019. Fuente. Elaboración propia.

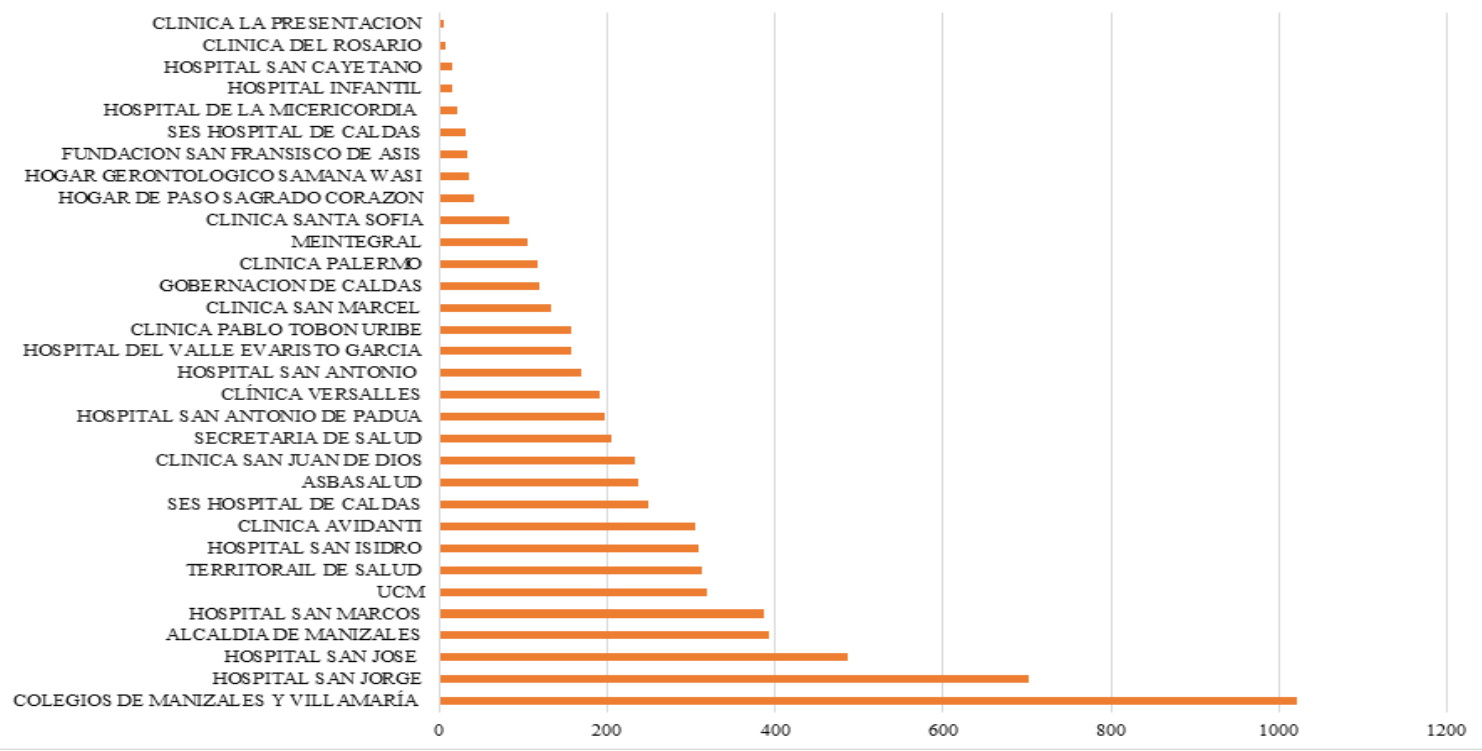


Rol del estudiante en los escenarios de práctica y beneficios de las instituciones...

Esto no significa que sea menos importante el plan de mejoramiento que se haya realizado en las IPS, puesto que éstos van dirigidos a la población interna y corresponden a los pacientes quienes se beneficias de educación en cambio de há- bitos de vida nocivos, y en la atención de salud que tan importantes para asegurar la correcta atención del usuario.

En referencia a la identificación de los grupos etarios y especiales que se han abordado en las IPS (Figura 4):

Figura 4. Grupos etarios y especiales beneficiarios en las prácticas formativas, elaboradas por estudiantes de VII semestre de enfermería. Universidad Católica de Manizales. 2017-2019. Fuente. Elaboración propia.

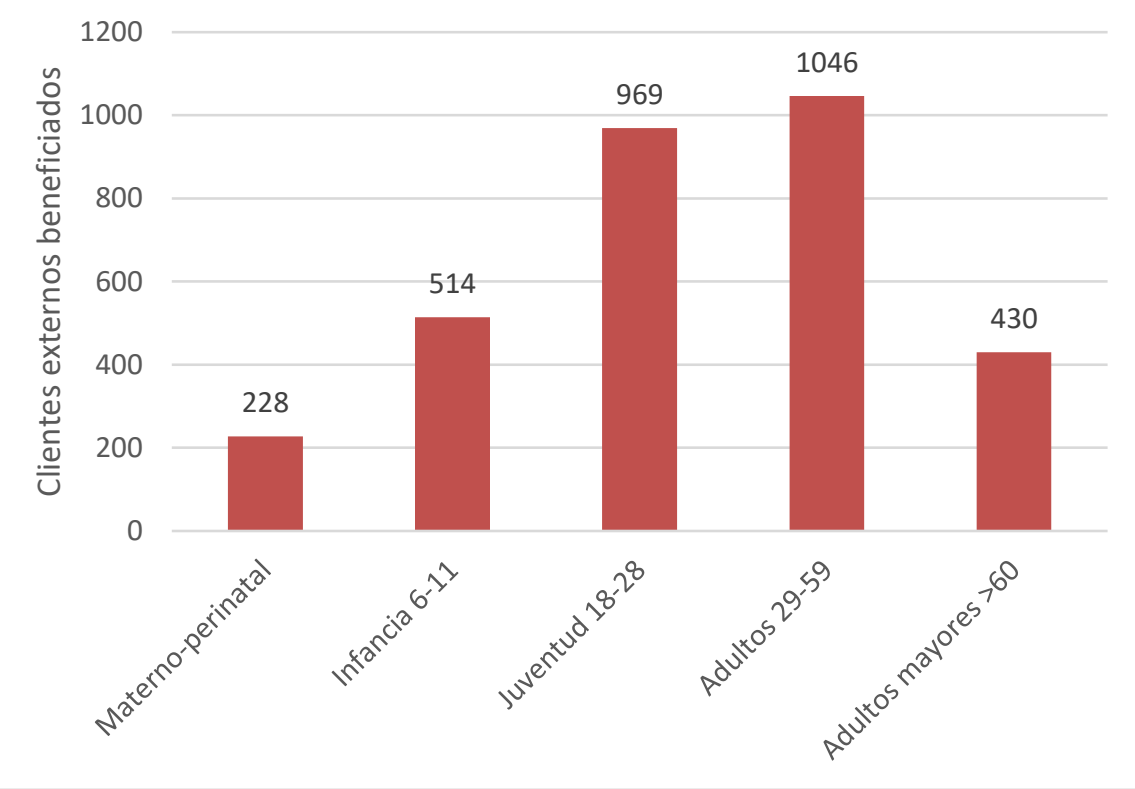

En esta Figura se muestran los principales grupos que se han abordado en los escenarios de práctica, beneficiados en actividades asistenciales, administrativas y educativas, observando que los grupos de mayor beneficio fueron el grupo de adultos, seguido de jóvenes, adultos mayores, infancia y materno-perinatal, respectivamente.

Con respecto a los temas y aspectos que se abordan por cada uno de esto grupos, se encuentra la información presentada en la Tabla 2.

Con respecto a la información presentada en la Tabla 2, se llama la atención sobre los siguientes aspectos: 
Tabla 2. Temas abordados por grupo etario y especial en actividades elaboradas por estudiantes de VII semestre de enfermería. Universidad Católica de Manizales. 20172019. Fuente. Elaboración propia.

\begin{tabular}{|c|c|c|}
\hline Grupo & Temas & $\begin{array}{c}\text { Número de pacientes } \\
\text { beneficiados }\end{array}$ \\
\hline \multirow{8}{*}{ Materno-perinatal } & Guía de extracción de leche materna & 40 \\
\hline & Vacunación para gestantes & 40 \\
\hline & Preparación para el parto & 35 \\
\hline & $\begin{array}{c}\text { Signos y síntomas de alarma en el } \\
\text { embarazo }\end{array}$ & 30 \\
\hline & Cuidados del recién nacido & 25 \\
\hline & Lactancia materna & 25 \\
\hline & Cuidado posparto & 18 \\
\hline & Estimulación intrauterina & 15 \\
\hline \multirow{7}{*}{$\begin{array}{l}\text { Infancia (6 - } 11 \\
\text { años) }\end{array}$} & Actividades recreativas & 266 \\
\hline & Dengue & 54 \\
\hline & Enfermedad diarreica aguda & 54 \\
\hline & AIEPI comunitario & 50 \\
\hline & Desparasitación masiva antihelmíntica & 35 \\
\hline & Enfermedad respiratoria & 30 \\
\hline & Salud oral & 25 \\
\hline \multirow{8}{*}{$\begin{array}{c}\text { Juventud }(18-28 \\
\text { años) }\end{array}$} & Infecciones de transmisión sexual & 213 \\
\hline & Alimentación saludable & 193 \\
\hline & Consumo de sustancias psicoactivas (SPA) & 165 \\
\hline & Embarazo en adolescentes & 135 \\
\hline & Planificación familiar & 115 \\
\hline & Complicaciones del consumo de SPA & 60 \\
\hline & $\begin{array}{l}\text { Prevención de enfermedades transmisibles } \\
\text { y no transmisibles }\end{array}$ & 53 \\
\hline & Hábitos de vida saludables & 35 \\
\hline \multirow{8}{*}{$\begin{array}{c}\text { Adultos }(29-59 \\
\text { años })\end{array}$} & Autoexamen de mama y citología & 159 \\
\hline & Cáncer de mama & 158 \\
\hline & Cáncer de cuello uterino & 127 \\
\hline & Autoexamen de testículo & 121 \\
\hline & Cáncer de próstata & 120 \\
\hline & $\begin{array}{l}\text { Sensibilización sobre la importancia de las } \\
\text { vacunas }\end{array}$ & 65 \\
\hline & $\begin{array}{c}\text { Estrategia CAPS (Comunidades con } \\
\text { autocuidado promotoras de salud) }\end{array}$ & 46 \\
\hline & Hábitos de vida saludables & 35 \\
\hline
\end{tabular}


Rol del estudiante en los escenarios de práctica y beneficios de las instituciones...

\begin{tabular}{|c|c|c|}
\hline & $\begin{array}{c}\text { Promoción y prevención en trabajadoras } \\
\text { sexuales }\end{array}$ & 33 \\
\hline & Enfermedad coronaria & 31 \\
\hline & Autocuidado de la salud & 30 \\
\hline & Salud oral & 27 \\
\hline & Estimulación intrauterina & 27 \\
\hline & $\begin{array}{c}\text { Educación en vacunación para gestantes y } \\
\text { menores de } 5 \text { años }\end{array}$ & 26 \\
\hline & Enfermedad mental & 21 \\
\hline & APS (Atención Primaria en Salud) & 20 \\
\hline \multirow{9}{*}{$\begin{array}{l}\text { Adultos Mayores } \\
\text { (más de } 60 \text { años) }\end{array}$} & Hipertensión arterial & 90 \\
\hline & Salud visual y auditiva & 74 \\
\hline & Vacunación en adulto mayor & 50 \\
\hline & Riesgo cardiovascular & 45 \\
\hline & Alteraciones en el adulto mayor & 39 \\
\hline & Salud oral & 37 \\
\hline & Enfermedad crónica & 35 \\
\hline & $\begin{array}{l}\text { Estrategia CAPS (Comunidades con } \\
\text { autocuidado promotoras de salud) }\end{array}$ & 32 \\
\hline & Hábitos de vida saludable & 28 \\
\hline
\end{tabular}

- Grupo materno perinatal: Las actividades realizadas con este grupo dan cuenta de temáticas propias de atención en el programa la de la madre y el recién nacido, que es un grupo de gran relevancia para las políticas nacionales. Se desarrollaron actividades asistenciales, administrativas y educativas en promoción y mantenimiento de la salud. Los tópicos de vacunación y extracción de leche materna, son aspectos abordados en los controles de enfermería que son transversales hasta el nacimiento del menor. De igual manera, la lactancia materna y la estimulación del recién nacido siguen posteriores al nacimiento, pero hacen parte de todas las acciones que deben realizar el personal de salud para garantizar la atención segura de este grupo.

- Grupo de 6 a 11 años, definido como infancia. El primer componente relevante son las actividades recreativas (266 personas beneficiadas) que aplica para el área de salud, especialmente mental, para estas edades. Las demás en su orden tienen que ver con la estrategia AIEPI para la prevención de las enfermedades prevenibles en la infancia y las intervenciones en los programas que se desarrollan según el perfil epidemiológico de la región, que en Colombia está caracterizado por enfermedad diarreica y enfermedades respiratorias agudas.

- Grupo de 18 a 28 años, definido como juventud. Para este grupo, y en res- 
Álvarez Castaño N, Ocampo JV, Cadavid M

puesta a los diferentes programas definidos por las IPS, el tema priorizado fue Infecciones de transmisión sexual (ITS) con un total de 213 personas beneficiadas. Sin dejar de ser importantes los programas y actividades para abordar el embarazo en adolescentes (135), la planificación familiar (115), alimentación y hábitos saludables que en conjunto logró 228 personas beneficiadas.

- Grupo de 29 a 59 años, definido como personas adultas. Para este grupo la mayoría de actividades están enfocadas en enfermedades crónicas no transmisibles, resaltando el cáncer con más de 400 personas abordadas, para el cual se hizo énfasis en el autoexamen en hombres y mujeres como prueba tamiz y como actividad de promoción y prevención.

- Grupo de mayores de 60 años, definido como adultos mayores. Para este grupo los planes de mejoramiento realizados por los estudiantes abordaron aspectos en mantenimiento de la salud con énfasis en programas de hipertensión arterial y riesgo cardiovascular (135 personas beneficiadas), salud visual y auditiva (74), entre otros.

Según las Rutas Integrales de Atención en Salud (RIAS), que son una estrategia de trabajo en salud para Colombia, se entienden por grupos de riesgo las personas con factores que hacen que se enferme, pueden tener varios años de duración y estar influenciados por determinantes socioeconómicos más generales. El nivel de ingresos y el nivel educativo pueden influir por ejemplo en los hábitos alimentarios y en el consumo de alcohol, los cuales "interactúan a su vez con causas fisiológicas y fisiopatológicas como la tensión arterial, los niveles de colesterol y el metabolismo de la glucosa, para dar lugar a enfermedades como los accidentes cerebrovasculares o la cardiopatía coronaria"7.

Tomando el lineamiento de las RIAS, se encuentra como el trabajo realizado por los estudiantes de VII semestre de enfermería, articulan su actuar dentro de las IPS al cumplimiento y abordaje de los grupos definidos de riesgo, según las particularidades de cada uno de estos. Adicionalmente, se encuentra que las IPS en la medida que cambian las normas y la reglamentación en salud, deben reorientar sus acciones de trabajo, sus programas y las prioridades en la atención, lo que hace que el estudiante este actualizado permanentemente para poder apoyar estos procesos.

Con respecto al desarrollo de planes de mejoramiento relacionados a aspectos administrativos por parte de los estudiantes, se encuentra que los temas que con mayor frecuencia se abordan son: pausas activas, prevención de accidentes laborales, fortalecimiento de la cultura de seguridad del paciente en prevención de caídas, manejo de historias clínicas, manejo de estrés, protocolo para la correcta aplicación de la escala de Morse, protocolo para el uso de elementos de bioseguridad, socialización de derechos y deberes, campaña para la prevención de accidentes por riesgo biológico y protocolo de manejo de pacientes aislados, respectivamente; como se observa en la Figura 5.

De estos se evidencia como cinco están relacionados de manera directa con Seguridad y Salud en el Trabajo, que 
Rol del estudiante en los escenarios de práctica y beneficios de las instituciones...

fueron los que ocuparon las frecuencias: uno, dos, cinco, siete y nueve, por su parte los demás temas están relacionados a aspectos de seguridad del paciente y otros estándares de calidad en salud; siendo todos temas de alta importancia en la formación de los profesionales de la enfermería.

Los aspectos clínicos y/o asistenciales que se observan en las intervenciones realizadas por el grupo de estudiantes objeto de estudio en el presente proyecto van directamente relacionadas con el cuidado de las personas y también obedecen a protocolos de atención, cumplimiento y aseguramiento de la correcta atención, así como temas específicos en ulceras por presión, manejo de heridas, aislamiento, manejo de la inmovilización, administración de medicamentos y reacciones adversas; que son actividades pilares en el quehacer de la disciplina de enfermería. Esta información se puede observar en la Figura 6.

Figura 5. Planes de mejoramiento con actividades administrativas, elaborados por estudiantes de VII semestre de enfermería. Universidad Católica de Manizales. 2017-2019. Fuente. Elaboración propia.

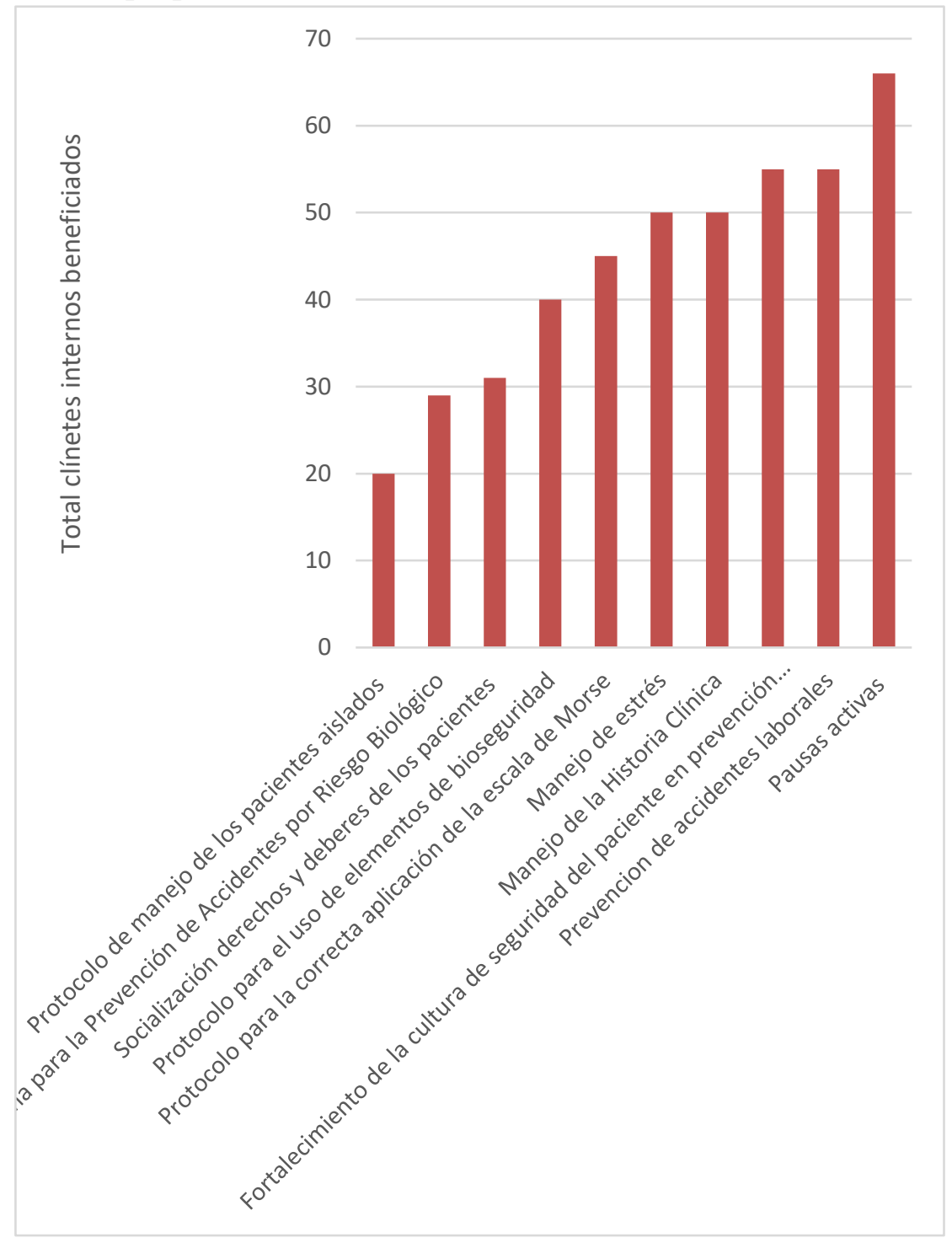


Figura 6. Planes de mejoramiento con actividades asistenciales, elaborados por estudiantes de VII semestre de enfermería. Universidad Católica de Manizales. 20172019. Fuente. Elaboración propia.

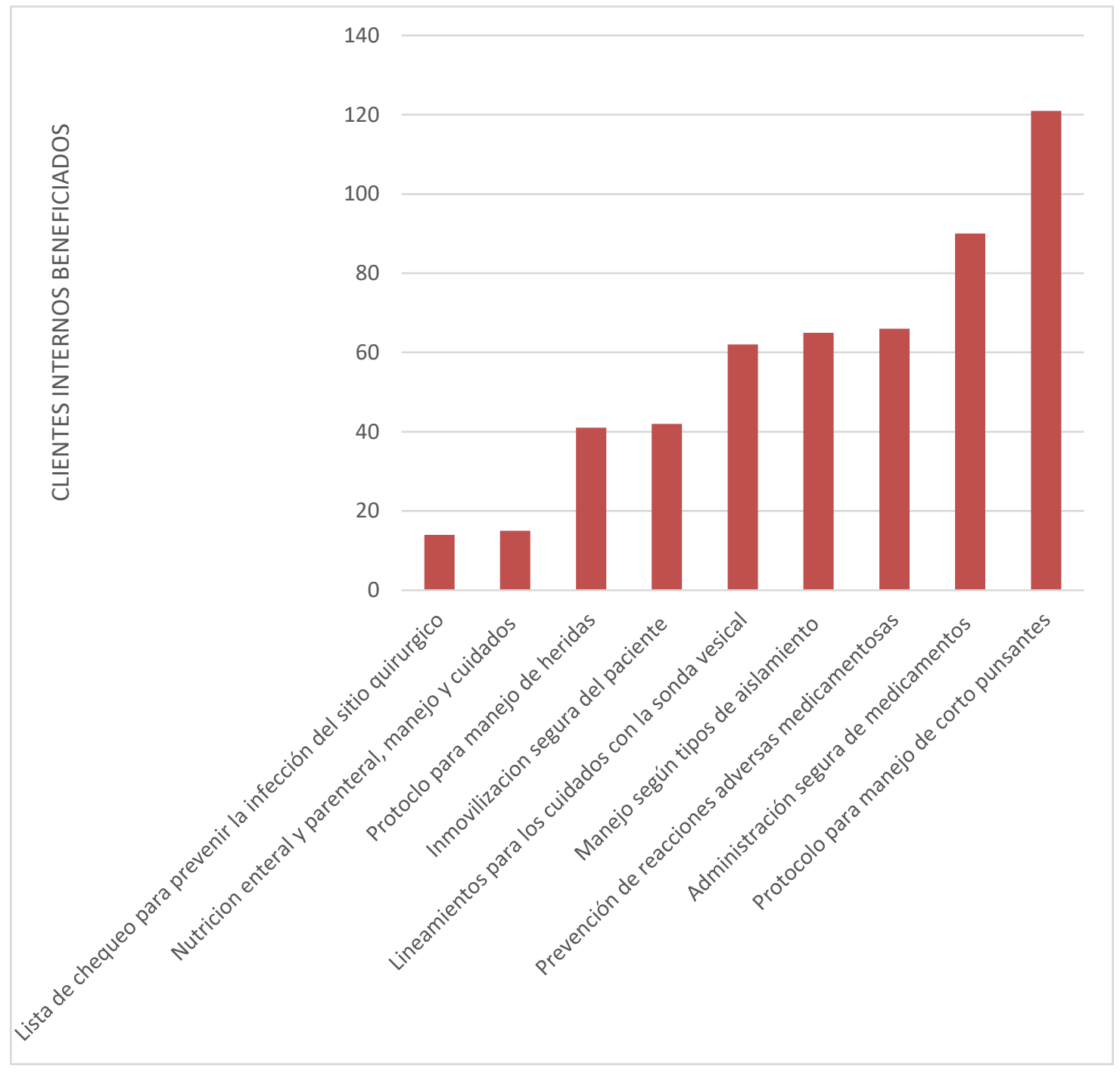

Al hacer particular énfasis en el tema de seguridad del paciente como política institucional, y en específico cuando se remite a los paquetes instruccionales del Ministerio de Salud y Protección Social relacionado a este tema, por parte de los estudiantes de enfermería se desarrollan en las IPS actividades específicas en prácticas seguras dentro de los planes de mejoramiento, es así como las practicas seguras desarrolladas han sido: identificación correcta del paciente y los laboratorios, prevención de úlceras por presión, seguridad en el quirófano y prevención de infecciones asociadas a la atención, como se observa en la Figura 7. 
Rol del estudiante en los escenarios de práctica y beneficios de las instituciones...

Figura 7. Planes de mejoramiento que involucran políticas institucionales de seguridad del paciente, elaborados por estudiantes de VII semestre de enfermería. Universidad Católica de Manizales. 2017-2019. Fuente. Elaboración propia.

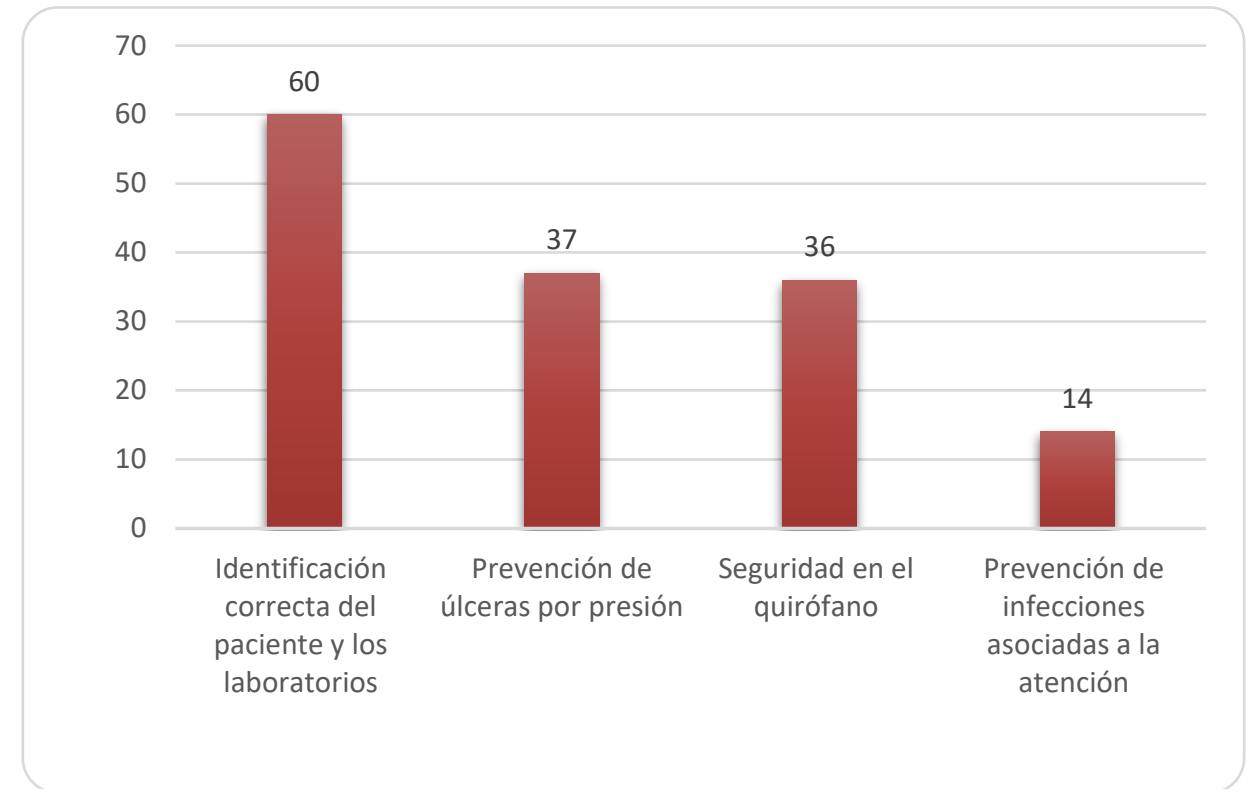

Como último aspecto relevante que se analiza en el presente proyecto del papel que los estudiantes juegan dentro de las IPS en las que realizan sus prácticas, está el relacionado a actividades de información, capacitación y educación, tanto a clientes internos como externos, que tienen un papel fundamental en la retroalimentación de los trabajadores y la educación de la persona de cuidado y su familia. Es así como en el periodo de 2017 a 2019 para este grupo de estudiantes se encontró una dedicación de 450 horas a este tipo de actividades, de las cuales 250 fueron dedicadas a los clientes externos y las restantes 200 horas a clientes internos. En la Tabla 3 se muestran los temas y el número de beneficiados de estas actividades.

Tabla 3. Temas y beneficiarios de actividades de capacitación elaboradas por estudiantes de VII semestre de enfermería. Universidad Católica de Manizales. 2017-2019. Fuente. Elaboración propia.

\begin{tabular}{|c|c|c|}
\hline \multirow{3}{*}{ Tipo de cliente } & Tema & $\begin{array}{c}\text { Número de } \\
\text { personas } \\
\text { beneficiadas }\end{array}$ \\
\hline \multirow{3}{*}{ Interno } & Lavado de manos & 1477 \\
\cline { 2 - 3 } & Disposición de residuos & 191 \\
\cline { 2 - 3 } & Prevención de flebitis & 80 \\
\cline { 2 - 3 } & Cuidados con la sonda vesical & 70 \\
\cline { 2 - 3 } & Socialización de los 10 correctos & 65 \\
\hline
\end{tabular}


Álvarez Castaño N, Ocampo JV, Cadavid M

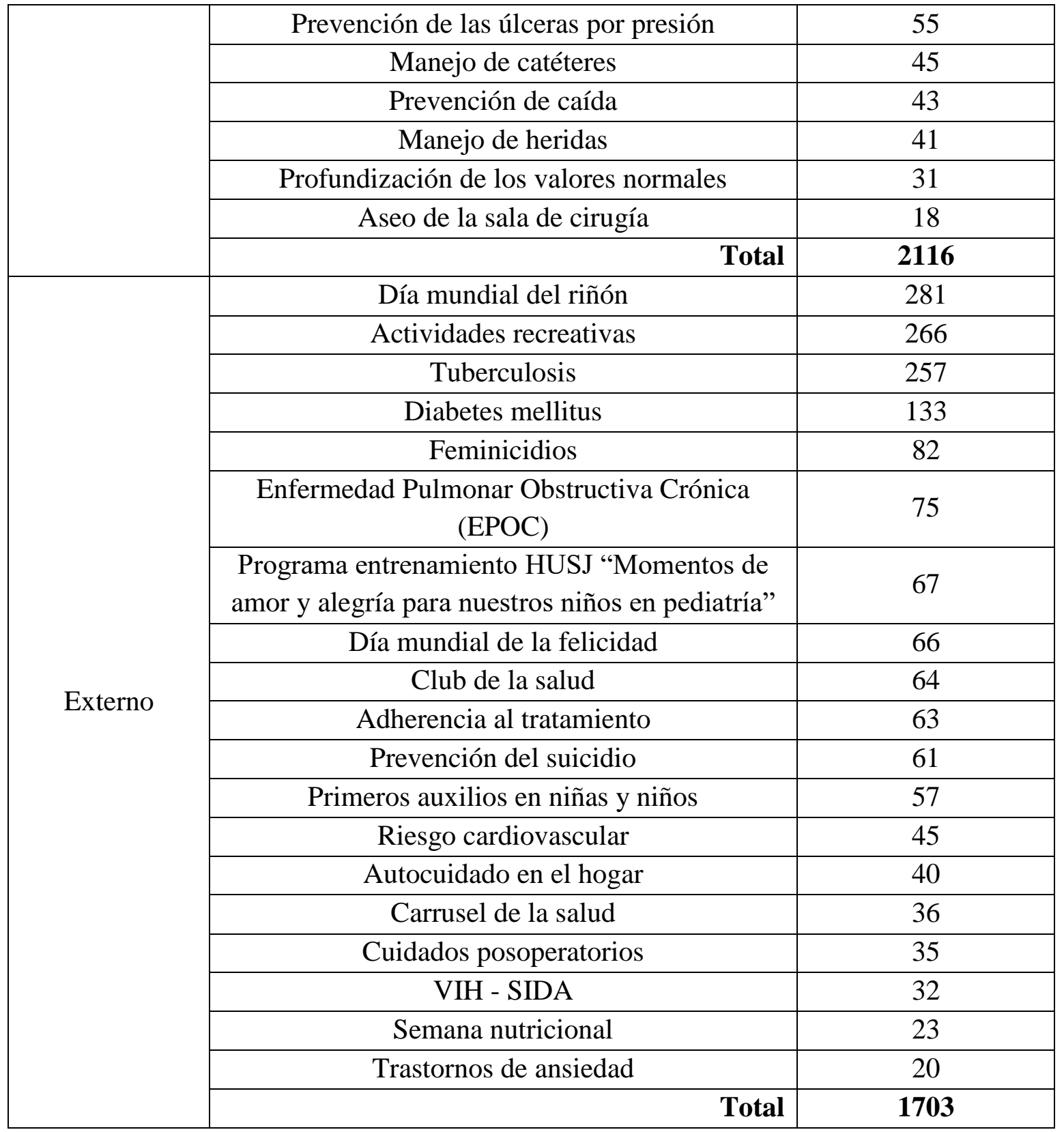

Las capacitaciones realizadas por los estudiantes que están involucradas en los planes de mejoramiento que benefician a las instituciones, tienen que ver con todas las acciones para potenciar las prácticas seguras, enfocadas en la seguridad de paciente, en las actividades para evitar infecciones asociadas a la atención de salud, obviamente todas dentro del campo disciplinar. El lavado de manos sigue siendo aquí y en todo el mundo la práctica 2021. HorizEnferm, 32,2,155-176 segura más trabajada frente a capacitación y retroalimentación al personal de salud de las IPS.

A lo largo de la presentación de los resultados, se puede observar el rol que el estudiante de enfermería de VII semestre tiene en los diferentes escenarios de práctica desde lo asistencial, lo administrativo y la promoción de la salud, que les permite trabajar los planes de mejoramiento de manera integral. Se 
Rol del estudiante en los escenarios de práctica y beneficios de las instituciones...

puede percibir la habilidad para la gestión administrativa, en las actividades propias del proceso enfermero de valoración, diagnóstico y entrega de cuidados integrales a la persona, familia y comunicad en beneficio de la salud a nivel individual y desde la perspectiva institucional a través de la IPS.

Se observa como el estudiante es capaz de liderar procesos, participar en la solución a los problemas, llevar a cabo procesos de seguimiento y control, abordar al cliente interno y externo, potenciar sus habilidades comunicativas, tomar decisiones y mostrar seguridad en el desarrollo de las actividades realizadas.

Por otro lado, y no menos importante, es ver el desempeño del estudiante de enfermería al integrar la familia y la comunidad al proceso de promoción y mantenimiento de la salud, recuperación y rehabilitación para posibilitar su adaptación al medio familiar, profesional y social

\section{DISCUSIÓN}

Mediante la recolección de la información se evidenció que el estudiante de enfermería planea actividades administrativas de acuerdo con las necesidades detectadas y en conjunto con el líder del proceso, lo que permite el desarrollo de una competencia básica enfocada a la solución de problemas de acuerdo a un contexto organizacional de la IPS. Los beneficios están enfocados en el control y seguimiento a los programas y políticas establecidas en las IPS. Adicionalmente el estudiante tiene la oportunidad de informar y educar al cliente interno en procesos prioritarios, reforzar la aplicación de protocolos para garantizar una óptima atención, apoya además el logro de objetivos en los programas, indicadores de gestión, puntos claves definidos en la habilitación o acreditación de las instituciones de acuerdo a lineamientos del Ministerio de Salud y Protección Social.

En el área asistencial el estudiante demostró su participación en el fortalecimiento de las prácticas seguras entre las cuales se encuentra la aplicación de los 10 correctos en administración de medicamentos, manejo y aplicación de las escalas de riesgo, manejo de flebitis y ulceras por presión, manejo adecuado de las heridas, control en la aplicación y adherencia a protocolos, entre otros; aunque fue más frecuente el lavado de manos. De esta manera se permite articular a lo planteado por Peñas et al., en el estudio de Seguridad del paciente en las prácticas formativas de un programa de pregrado en Terapia Ocupacional, en el que se realizó una encuesta a estudiantes de este pregrado sobre atención segura de usuarios, en la cual se identificó el incidente de caídas como el que se reporta en menor medida, esto se debe al compromiso ético que debe prevalecer por la seguridad del usuario ${ }^{8}$.

También se observa la participación en las consultas de enfermería, acciones de educación y actividades de promoción y prevención en los programas para gestantes, prevención del cáncer, consulta a los adolescentes, programa de vacunación y atención de enfermedades crónicas no transmisibles del adulto mayor; de la misma manera, Gerbotto et al., describen la representación de estudiantes de enfermería en torno al rol enfermero, en el cual se realizaron entrevistas estructuradas en la que se 
diferenció la mirada del rol enfermero de los estudiantes antes de ingresar a la universidad, en la cual sus percepciones se centran en torno a las tareas asistenciales como de higiene y confort, pero durante su estancia en la universidad, dan una mirada más amplia a lo que es el rol de enfermería viendo al paciente como ser integral, el cuidado que puedes brindar, la promoción y la prevención siendo el enfermero el contacto más directo con el paciente ${ }^{9}$.

Adicionalmente, se logró encontrar que algunos procesos no estuvieron intervenidos y beneficiados por completo, pero esto tiene mucha relación con los intereses y las necesidades de las instituciones; las evidencias que se muestran en este estudio reflejan los procesos abordados según lo dispuesto organizacionalmente, los tópicos más relevantes fueron seguridad del paciente, calidad y acciones en los programas vigentes en salud según curso de vida. Se percibe que existe un trabajo organizacional conjunto, que lleva a cambios positivos en las instituciones prestadoras de servicios, ya que los estudiantes realizan intervenciones priorizadas para reforzar habilidades, actitudes y prácticas en el cliente interno y en mantenimiento de la salud, con los clientes externos para mejorar sus condiciones de salud. Con la finalidad que las actividades realizadas por los estudiantes en los diferentes ámbitos de cuidado, sean suficientes y eficaces, requiere que las instituciones apliquen las acciones de manera continua, y es necesario consolidar una cultura institucional educativa y no punitiva que parta del informe y análisis entregado en cada uno de los planes de mejoramiento.
Álvarez Castaño N, Ocampo JV, Cadavid M

\section{CONCLUSIONES}

El rol del estudiante de enfermería de VII semestre de la Universidad Católica de Manizales, en Colombia, se concibe como protagonista de un proceso de aprendizaje continuo en un escenario académico y científico ofrecido por las Instituciones Prestadoras de Servicios de Salud (IPS) apalancado en las funciones que se les otorga y en las actividades delegadas por los profesionales que los acompañan, lo que permite la construcción de conocimiento práctico sobre la enfermedad humana, su manejo y su prevención, con la finalidad de generar pensamiento crítico sobre él.

De esta manera se constituye un trabajo organizacional conjunto entre la institución universitaria y la IPS, que lleva a cambios positivos en éstas, ya que los estudiantes realizan intervenciones priorizadas para reforzar habilidades, actitudes y prácticas en el cliente interno y en mantenimiento de la salud con los clientes externos para mejorar sus condiciones de salud.

Es así como se identifica que el rol que desempeñan los estudiantes de VII semestre, es de gran importancia para apoyar y apalancar procesos administrativos, clínicos, de promoción y mantenimiento de la salud en las IPS, debido a que permiten incrementar la fuerza de respuesta ante cada una de estas áreas sin costo a la nómina de la institución, además ofrece la oportunidad para aprovechar este talento humano en beneficio de educación para cliente interno y externo, identificación de problemas y según los resultados, diseñar y hacer seguimiento a los procesos que en su momento se observan como críticos. 
Rol del estudiante en los escenarios de práctica y beneficios de las instituciones...

\section{REFERENCIAS BIBLIOGRÁFICAS}

1. Figueiredo Mirela López de, D' Innocenzo M. Adverse events related to pratical assistence: an integrative review. Enferm. glob. [Internet]. 2017 [cited 2021 July 06]; 16(47): 605-650. Available

from: http://scielo.isciii.es/scielo.php?script= sci_arttext\&pid=S1695-

61412017000300605\&lng=en. Epub July 01,2017. https://dx.doi.org/10.6018/eglobal.16.3 .256091

2. Tirado Otálvaro AF, Lopera Castro G, López Montoya B, Londoño Arboleda C, Echeverri Tamayo C. Diez años de logros del programa de Enfermería de la Universidad Pontificia Bolivariana en comunidades vulnerables de Medellín y el área metropolitana. Med UPB [Internet]. 15 de diciembre de 2012 [citado 6 de julio de 2021]; 31(2):160-9. Disponible en: https://revistas.upb.edu.co/index.php/ medicina/article/view/1694

3. Mármol López María Isabel, Miguel Montoya Isabel, Montejano Lozoya Raimunda, Escribano Pérez Almudena, Gea Caballero Vicente, Hontangas Antonio Ruiz. Impacto de las intervenciones enfermeras en la atención a la cronicidad en España. Revisión sistemática. Rev. Esp. Salud Pública [Internet]. 2018 [citado 2021 Jul 06]; 92: e201806032. Disponible en:

http://scielo.isciii.es/scielo.php?script= sci_arttext\&pid=S1135-

57272018000100501\&lng=es. Epub 27-Jun-2018.
4. Escobar MP, Mejía AM, Betancur SI. Cambios en el autocuidado de estudiantes colombianos de enfermería, durante la formación profesional. 2015. Hacia promoc. salud. 2017; 22(1): $27-$ 42. DOI: $10.17151 / \mathrm{hpsal} .2017 .22 .1 .3$

5. Guerrero-Ramírez R, Meneses-La Riva ME, De La Cruz-Ruiz M. Cuidado humanizado de enfermería según la teoría de Jean Watson, servicio de medicina del Hospital Daniel Alcides Carrión. Lima- Callao, 2015. RENH [Internet]. 20feb.2017 [citado 6jul.2021]; 9(2):133. Available from: https://revistas.upch.edu.pe/index.php/ RENH/article/view/3017

6. Colombia. Ministerio de Salud y Protección Social. (NaN). Política de Atención Integral en Salud "Un sistema de salud al servicio de la gente". Observatorio Nacional de Salud Mental- ONSM; Documentos técnicos REPS; Observatorio Nacional de Cáncer- ONC; Política integral de Atención en salud; Observatorio Nacional de Salud Mental- ONSM; 97 p. Recuperado de: https://www.minsalud.gov.co/sites/rid/ Lists/BibliotecaDigital/RIDE/DE/mod elo-pais-2016.pdf

7. Colombia. Ministerio de Salud y Protección Social. (2016). RIAS para población con riesgo o alteraciones cardio -cerebro-vascular-metabólicas manifiestas, y las RIAS específicas para Hipertensión arterial- HTA, Diabetes mellitus - DM y Obesidad. Política integral de Atención en salud; 1 carpeta comprimida en zip Recuperado de: https://www.minsalud.gov.co/sites/rid/ 
Álvarez Castaño N, Ocampo JV, Cadavid M

Lists/BibliotecaDigital/RIDE/VS/riascardio-cerebro-vascular.zip

8. Peñas-Felizzola Olga L., ParraEsquivel Eliana I., Gómez-Galindo Ana M. Seguridad del paciente en las prácticas formativas de un programa de pregrado en Terapia Ocupacional. Rev. Salud pública [Internet]. 2017 Aug [cited 2021 July 06]; 19 (4): 446-452. Available from: http://www.scielo.org.co/scielo.php?sc ript=sci_arttext\&pid=S012400642017000400446\&lng=en. https://doi.org/10.15446/rsap.v19n4.61 737.

9. Gerbotto M, Saez NB, Gattari M de los Ángeles, Daro MV. Prácticas y representaciones de estudiantes de enfermería en torno al rol enfermero. RUE [Internet]. 11 de agosto de 2019 [citado 6 de julio de 2021]; 14(1). Disponible en: https://rue.fenf.edu.uy/index.php/rue/ar ticle/view/268

10. (2007). Herramientas para promover la estrategia de la seguridad del paciente en el sistema obligatorio de garantía de calidad de la atención en salud. El Ministerio, Recuperado de https://www.minsalud.gov.co/sites/rid/ Lists/BibliotecaDigital/RIDE/VS/PSA/ herramientas-seguridad-paciente.pdf

11. Gualdrón M, Barrera-Ortega K, ParraGonzález N. Percepción de la enseñanza en cuidado humanizado vs la práctica formativa por estudiantes de enfermería. aibi [Internet]. 1 de enero de 2019 [citado 6 de julio de 2021]; 7(S1):7-12. Disponible en: https://revistas.udes.edu.co/aibi/article/ view/1680.
12. Colombia. Ministerio de la Protección Social \& Universidad CES. (2009). Promoción de la cultura de seguridad del paciente. Seguridad del Paciente; Calidad de la Atención en Salud; Seguridad del Paciente; 51p. Recuperado de: https://www.minsalud.gov.co/sites/rid/ Lists/BibliotecaDigital/RIDE/DE/CA/ Guia_promocion_seguridad_paciente. pdf

13. (2008). Lineamientos para la implementación de la política de seguridad del paciente. Seguridad del paciente; Calidad de la atención en salud; Seguridad del paciente. 52 p. Recuperado de: https://www.minsalud.gov.co/sites/rid/ Lists/BibliotecaDigital/RIDE/DE/CA/ LINEAMIENTOS_IMPLEMENTACI ON_POLITICA_SEGURIDAD_DEL_ PACIENTE.pdf

14. Campiño-Valderrama Sandra Milena, Duque Paula Andrea, Cardozo Víctor Hugo. Percepción del paciente hospitalizado sobre el cuidado brindado por estudiantes de enfermería. Univ. Salud [Internet]. 2019 Dec [cited 2021 July 06]; 21(3): 215-225. Available from:

http://www.scielo.org.co/scielo.php?sc ript=sci_arttext\&pid=S0124$71072019000300215 \& \operatorname{lng}=$ en. https://doi.org/10.22267/rus.192103.15 8

15. Guía metodológica. Observatorio nacional de calidad de la atención en salud. Ministerio de Salud y Protección Social. Bogotá D.C, Colombia. [INTERNET] 2016. (citado el 14 de abril de 2020). Disponible en: 
Rol del estudiante en los escenarios de práctica y beneficios de las instituciones...

https://www.minsalud.gov.co/sites/rid/

Lists/BibliotecaDigital/RIDE/DE/CA/

Guia-metodologica-observatorio-

calidad.pdf Ministerio de la Protección

Social. Calidad en salud en Colombia

Los principios Programa de Apoyo a la

Reforma de Salud. 2018 [Internet].

[Disponible el 16 abr 2020]Disponible

en:

https://www.minsalud.gov.co/Docume ntos $\% 20 y \% 20$ Publicaciones/CALIDA D\%20EN\%20SALUD\%20EN\%20CO

\section{LOMBIA.pdf}

16. Yáñez, César Enrique Jiménez, et al. "Aprender a hacer: la importancia de las prácticas profesionales docentes." Educere (internet) 18.61 (2014): 429438. [citado el 16 de abril de 2020]. Disponible en: http://www.redalyc.org/articulo.oa?id= 35639776005

17. Olivas-Peñúñuri, Ma Remedios, et al. "Acompañamiento de pares en la práctica clínica de enfermería como estrategia para la mejora del cuidado de la salud." Boletín Clínico Hospital Infantil del Estado de Sonora. 2017 (internet) $34.1 \quad$ (2017): 20-25. [Internet]. [Bol Clin Hosp Infant Edo Son 2017; 34(1)] [citado el 16 de abril de 2020].

18. Pérez Pino Michel, Enrique Clavero José Osvaldo, Carbó Ayala José Eugenio, González Falcón Marisol. La evaluación formativa en el proceso enseñanza aprendizaje. EDUMECENTRO [Internet]. (2017). Sep. [citado 2021 Jul 06]; 9(3): 263283. Disponible en: http://scielo.sld.cu/scielo.php?script=s ci_arttext\&pid=S207728742017000300017\&lng=es.

19. González C. VE. La calidad de las prácticas formativas del área de la salud: la intersección de dos sectores. Una experiencia desde la Escuela de Microbiología de la Universidad de Antioquia. Hech Microb. [Internet] 2014 [citado 6 de julio de 2021]; 3(2):73-9. Disponible en: https://revistas.udea.edu.co/index.php/ $\mathrm{hm} /$ article/view/18739

20. Álvarez-Álvarez C. El reto de la fundamentación científica de la formación del profesorado. Tarbiya [Internet]. 10 de diciembre de 2017 [citado 6 de julio de 2021];(45). Disponible en: https://revistas.uam.es/tarbiya/article/v iew/7587

21. Marcolino, Taís Quevedo, Lourenço, Gerusa Ferreira e Reali, Aline Maria de Medeiros Rodrigues"Isso eu levo para a vida!": aprendizagem da prática profissional em uma Comunidade de Prática. (internet) 2017 [citado el 6 Julio 2021] Disponible en: https://doi.org/10.1590/1807-

57622016.0099

22. Cedeño Sánchez, L.V., \& Santos Naranjo, J. La práctica pre-profesional: vía para la formación profesional integral desde sus incidencias en la investigación. Revista Universidad y sociedad. (Internet) 2017 [Citado el 13 de nov 2019] Disponible en: http://scielo.sld.cu/pdf/rus/v9n3/rus163 17.pdf 